\title{
Ubiquitous and Comprehensive Healthcare: Expanding Technologies and Systems to Enable New Delivery Models
}

\author{
Benjamin Schooley, MBA, PhD \\ College of Engineering and Computing \\ University of South Carolina \\ Bschooley@cec.sc.edu \\ Nitin Patel, MD \\ School of Medicine \\ University of South Carolina \\ Nitin.Patel@prismahealth.org
}

\author{
Sue Feldman, RN, MEd, PhD \\ School of Health Professions \\ University of Alabama at Birmingham \\ Sfeldman@uab.edu \\ Saif Khairat, PhD \\ School of Nursing \\ University of North Carolina at Chapel Hill \\ saif@unc.edu
}

\begin{abstract}
Whether at home, work, school, or traveling abroad, healthcare is in demand outside the walls of traditional healthcare facilities. Rapidly changing delivery models are shaping the new healthcare landscape, which has only accelerated because of the COVID-19 worldwide pandemic. The papers in this minitrack highlight human needs, societal problems (e.g., racism), and government vs individual control factors related to data sharing that must be confronted more directly in order to enable new delivery models for ubiquitous and comprehensive healthcare.
\end{abstract}

\section{Introduction}

When we first proposed this minitrack last February, we had no idea the havoc that would result from a COVID-19 outbreak that lurked on the horizon. We did not see what would happen in the months ahead - how a worldwide pandemic would force changes to healthcare delivery. We witnessed our respective healthcare systems in the Carolinas and Alabama implement COVID-19 chatbots, deploy exposure notification apps that live on our phones, and expand telehealth visits to most primary care and many specialty care appointments. Telehealth and remote patient monitoring technology implementations and use seems to have expanded at lightspeed, reaching levels that many researchers and technologists believe should have been reached a decade ago. These current realities fit well with this minitrack theme - though ironically (yet not surprisingly due to many unforeseen challenges) only a few articles made their way through the review process.
Our motivation for this minitrack was our collective experience in research and practice over the last three decades. We collectively had observed innovations come and go with mild to moderate effects on healthcare practice. Certainly, we've seen the effects pick up steam over the last decade and become more far reaching and impactful. For example, telehealth services and supporting technologies have expanded into new domains, remote patient monitoring has become common practice for certain conditions, patient engagement has become more empowering and meaningful to some patients and providers due to patient facing and consumer facing apps, and augmented intelligence (AI) and machine learning have gained significant ground. Our forward-thinking mantra was that health happens everywhere, though healthcare has traditionally been contained to predefined environments. Whether at home, work, school, or traveling abroad, healthcare is in demand outside the walls of traditional healthcare facilities. A wide range of organizations (e.g., pharmacies, retailers, tech firms, large employers) are filling gaps in the marketplace that current care delivery providers are not meeting. Such examples are functional medicine enterprises, at-home diagnostic kits, convenient on-premise-on-demand primary care, and synchronous or asynchronous virtual visits. Rapidly changing delivery models are shaping the new healthcare landscape. Again, COVID-19 has forced major changes in these areas.

A key focus of research and innovation in healthcare should be on the ever-changing nature of business, financial and care delivery models and the role of information systems and technology (IS\&T) as enablers. Innovation research highlights novel research on emergent digital health information systems and 
technologies, including their design, field testing, evaluation, and broader impacts. Researchers are creating new and innovative models, frameworks, and technologies that delve into how IS\&T enables and supports health to happen everywhere. Resulting technology artifacts include mobile devices, wearables and other IOT devices, sensors, telehealth applications, tele-monitoring, mHealth apps, EHR extension apps for non-traditional healthcare environments, and integrations with these technologies and EHRs. We propose that more applied research is needed to address how artifact designs, implementations, methodologies, and theories are affecting healthcare delivery models and challenging current models to improve access, patient engagement, costs, and population health.

The papers in this minitrack address the theme in two different but important ways. One confronts the need to reevaluate access to care for minorities, while the other presents data sovereignty and data donation cycles as a way to increase data availability for effective personalized healthcare. Both articles address interdisciplinary "systems" challenges with significant institutional, technological, and policy implications. The first article, by Paras Bhatt and colleagues, describes a cumulative and increasing gap in healthcare access and the resulting inequality which leads to Hispanics being negatively affected by the novel coronavirus more than any other race or ethnicity. Using empirical case comparisons (ECCs), the authors find that socioeconomic status alone does not explain higher COVID-19 infection rates among Hispanics. They propose a range of other factors that may help explain healthcare inequalities, including racism, superlative institutional factors, and mental health factors and apply cumulative inequality theory to address the gap. Their future work is promising, working to analyze publicly available data on COVID-19 with the help of machine learning models to gain insights about how technology can be used as a tool in the study of racial and ethnic disparities and inequalities in the US healthcare system.

The second paper, by Schinle and colleagues, also alludes to the current COVID-19 pandemic. In particular, the legal and ethical pressures it has placed on governments concerning the collection and use of digital health data for improved personalized and population health. Options include a range of government interventions vs voluntary participation from citizens, and compulsion or voluntariness in their use. Schinle and colleagues address structural, economical, medical and technological implications of self-determined medical data donation using a design science research methodology. The authors identify requirements for self-determined health data management to embed in a value-added data donation cycle, or patient data that is used, re-used, and incrementally increases during research and development. Their future work poses many challenges, including the self-determined control of decentralized health data flows and the privacy-preserving traceability of data analytics steps needed to support new delivery models.

These papers address large-scale interdisciplinary socio-technical issues where technology artifacts are indelibly intermixed with deeply embedded social issues. They support the notion that technology continues to advance toward ubiquitous and comprehensive healthcare. Yet, they also highlight that in order to enable new delivery models, socio-technical issues relating to data, human needs, societal problems (e.g., racism), and government vs individual control must be confronted more directly. 